\title{
A New Approach to Fracture Simulations: The Electromagnetic Breakable and Reusable Human Lower Limb Model
}

Geoffrey T Desmoulin', Fariha An-Nafee Rashid ${ }^{2}$, Colin Ropchan ${ }^{2}$, Jason Meitz ${ }^{2}$, Kunj Upadhyaya ${ }^{2}$ and Sammy Yan ${ }^{2}$

${ }^{1}$ GTD Engineering, 218-E4th Ave, Vancouver, BC, V5T-1G5, Canada

${ }^{2}$ Department of Mechanical and Manufacturing Engineering, University of Calgary, 2500 University Drive Northwest, Calgary, AB, T2N 1N4, Canada

\begin{abstract}
Background: Lower limb fractures are relatively common injuries resulting from sport or vehicle accidents, falls, and other situations. The most frequent site of such a fracture is the distal third of the tibia. Different methods ranging from crash tests using anthropometric dummies to finite element modelling have been used to study fracture mechanisms. This study developed an electromagnetic human lower limb model to provide a prototype for testing compound fractures at desired break locations under forces similar to that of a real human tibial fracture. The prototype's ability of simulating such a fracture was assessed.
\end{abstract}

Methods: The prototype had realistic dimensions, fracture locations and load to fracture magnitudes of a 50th percentile human male lower limb. Both the limb and the testing assembly were modeled in SolidWorks (Dassault Systems, SOLIDWORKS Corp, Massachusetts, USA). The electromagnetic model had a lower and upper segment with the electromagnet (APW Company, Rockway, New Jersey, USA) mounted in-between. A drop test was used to test the model dynamically.

Results: The model was tested under different loads and peak forces that led to a break were identified. The model was reusable after each break and is therefore suitable for repeated testing.

Conclusion: This paper presents the characteristics of the electromagnetic breakable and reusable human lower limb model, results of the break under similar forces that cause real-life human lower limb compound fractures, and recommendations for future development of the model.

Keywords: Fracture; Dynamics; Biomechanics; Tolerance; Prototype; Simulation

\section{Introduction}

Lower limb fractures are frequent results of injuries that can occur during a number of activities including sports, self-defense situations $[1,2]$, and military, para-military or violent encounters $[3,4]$. There is an anatomical region of the lower limb where fractures occur most frequently: the tibia. The tibia is a long hollow bone, which has a metaphysis and epiphysis at both sides of a tabular diaphysis, the shaft [5]. Fractures of the tibial shaft are common and share characteristics in terms of site, type and local fracture mechanisms [6]. Top tier self-defense students training for violent deadly force encounters are taught to place their kick on the lower limb at specific impact locations [7]. Figure 1 shows that strike placement occurs at approximately 30 degrees from the lateral-medial axis in the horizontal plane and at approximately 45 degrees from the lateral-medial axis in the frontal plane. The strike occurs slightly above the lateral or medial malleolus in the distal third region of the limb [7]. This strike location has been determined to be the weakest point of the tibia [8].

Furthermore, a study investigating lower limb fractures in pedestrians and cyclists after side impacts caused by passenger vehicles provided additional data that confirmed the general location of where fractures occur. Nearly $80 \%$ of the bumper heights were between 320 $460 \mathrm{~mm}$ above the ground level, which corresponds to the middle to proximal region of the lower limb, yet the majority of the fractures still occurred in the distal third region of the lower limb [9]. Also, observations of lower limb injuries during soccer (football) matches showed that complete fractures typically occured when the player was kicked in the lower limb and that the most frequent fractures occured in the distal third region of the lower limb [10]. Another study also confirmed that $88.9 \%$ of fractures caused by falls or during various sports and pedestrian collisions occured in the middle to distal third part of the tibial shaft [11].

Previously, Young's moduli and shear moduli of the tibial bone tissue were used to determine that while the whole bone fails under tension microscopically, macroscopically the tibia is most likely to fail under shear forces or bending rather than compression [12,13]. It was found that the tibial Young's modulus is isotropic along its horizontal planes [12] and the bending moment varies negligibly between anteriorposterior and lateral-medial directions [14].

Currently, medical professionals use plastic stand-ins or preclinical models to simulate compound fractures when training, whereas high-end self-defense practitioners use various types of hanging and hand-held heavy bags or static dummies for full speed and power training [7]. These devices have significant training limitations: a) force perturbations upon strike impact are relatively predictable [15];

b) while the practitioner's muscular power is affected by training, the reactivity of the training bag or dummy is not [16];

*Corresponding author: Geoffrey Thor Desmoulin, Ph.D., R. Kin. President and Senior Engineer at GTD Engineering 218-E4th Ave, Vancouver, BC, V5T-1G5, Canada, Tel: 1-604-842-4831; E-mail: gtdesmoulin@gtdengineering.com

Received February 18, 2014; Accepted May 15, 2015; Published May 23, 2015

Citation: Desmoulin GT, Rashid FAN, Ropchan C, Meitz J, Upadhyaya K, et al. (2015) A New Approach to Fracture Simulations: The Electromagnetic Breakable and Reusable Human Lower Limb Model. J Forensic Biomed 4: 121. doi: 10.4172/2090-2697.1000121

Copyright: ( 2015 Desmoulin GT, et al. This is an open-access article distributed under the terms of the Creative Commons Attribution License, which permits unrestricted use, distribution, and reproduction in any medium, provided the original author and source are credited. 


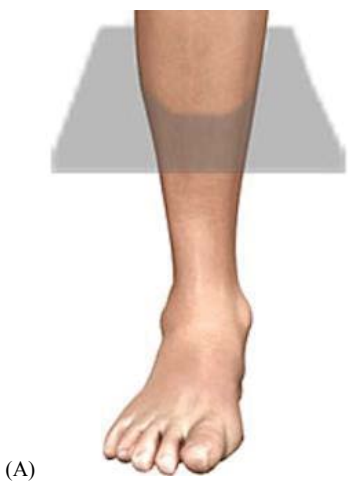

(B)

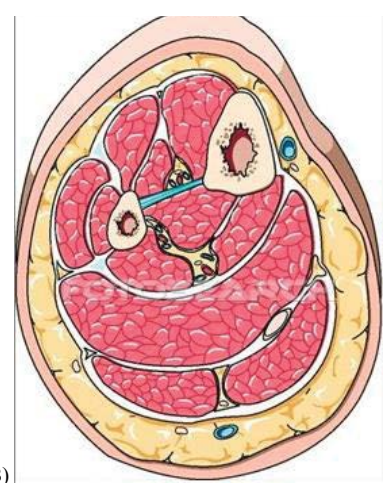

Figure 1: The anatomy of the tibia and fibula with approximate impact vectors in the frontal $(A)$ and horizontal $(B)$ planes. An anterior view of the lower limb $(A)$ indicating where the cross-section in (B) occurred. The arrows indicate the approximate planar impact in $2 \mathrm{D}$. The anterior view impact from the frontal plane (A) is approximately 45 degrees and the cross-section impact angle from the horizontal plane $(B)$ is approximately 30 degrees (concept from [7] Desmoulin and Larkin).

c) effects of the human geometry (shape) and tissue composition (stiff versus compliant) at impact are not replicated by the bag or dummy [7]; and

d) the load to cause injury is not experienced by the practitioner prior to critical incidents and therefore remains novel [7].

Lastly, the actual force that is required to break the human tibia under a specific strike remains unknown and difficult to calculate analytically due to the complexity of the human tissues involved and the high number of strike impact characteristics, such as the location, loading rate, strike technique and human anatomy used for strike.

Consequently, there is a demand for realistic fracture response testing and equipment among researchers including medical professionals and military, police or high-end self-defense institutions that are teaching techniques reserved for deadly force encounters in order to understand fracture dynamics in real-time. Therefore, a lower limb model on which realistic compound fractures could be tested in a similar time frame during which human fractures occur, was developed. A model with human male lower limb dimensions was created so that it would break under similar forces at which a corresponding $50^{\text {th }}$ percentile human male lower limb breaks. Dynamic tests were conducted to validate whether the model breaks at a desired location under a desired force. The aim of this paper was to provide detailed characteristics of the electromagnetic human lower limb model and the fractures that have been generated using it.

\section{Methods}

\section{Break location}

Based on the literature discussed previously it was decided to use the lower third part of the tibia for the compound fracture simulations [6-9]. It can be estimated that for a barefoot $50^{\text {th }}$ percentile male, the minimal circumference of the tibia is located approximately $132 \mathrm{~mm}$ above the ground using PeopleSize Software (Open Ergonomics Ltd., Loughborough, UK). In order to compensate for the height of the standard male shoe sole [17], $25 \mathrm{~mm}$ was added to the model. Therefore the total distance from the ground used in modeling and prototyping the limb was $157 \mathrm{~mm}$.

\begin{tabular}{|c|c|c|}
\hline & Electromagnetic model & $50^{\text {th }}$ percentile male \\
\hline Length & 496 & $496[20]^{\star *}$ \\
\hline Center of Gravity & 214 & $217[22]$ \\
\hline Mass & 5.4 & $3.9[20]$ \\
\hline Break location & 157 & 157 \\
\hline Diameter & 89 (without "skin") & 110 \\
\hline
\end{tabular}

* The length and diameter are in millimeters $(\mathrm{mm})$, the center of gravity and break location are in millimeters $(\mathrm{mm})$ from the ground, and the mass is in kilograms.

** $402 \mathrm{~mm}$ tibial length with $94 \mathrm{~mm}$ ankle condyles depth to the ground.

Table 1: The characteristics of the electromagnetic lower limb model compared to a 50 th percentile male data*

\section{Break force}

A literature review was conducted and simplifications, detailed below, were made to determine the approximate applied failure load required to cause the model to break. Firstly, the tibia was modeled as a beam as X-ray films from pedestrian-vehicle accident victims revealed that a typical tibial fracture pattern is similar to bending failures of brittle beams [18]. Secondly, the properties of the tibial structure as outlined in the introduction allowed the prototype to be modeled as a cylindrical beam under bending, regardless of its direction. In order to determine the Young's moduli in each direction, the inverse of the elastic stiffness matrix was used to obtain the elastic compliance matrix: $[\mathrm{s}]=[\mathrm{c}]^{-1}$. Based on this approach the values for the Young's moduli and shear moduli for tibial cortical bone were calculated. The calculated values for Young's moduli and shear moduli for tibial cortical bone were:

$$
\begin{aligned}
& \mathrm{E}_{\mathrm{xx}}=11.8 \mathrm{GPa} ; \mathrm{E}_{\mathrm{yy}}=12.2 \mathrm{GPa} ; \mathrm{E}_{z z}=20.8 \mathrm{GPa} \\
& \mathrm{G}_{\mathrm{yz}}=2.9 \mathrm{GPa} ; \mathrm{G}_{\mathrm{zx}}=2.6 \mathrm{GPa} ; \mathrm{G}_{\mathrm{xy}}=2.0 \mathrm{GPa}
\end{aligned}
$$

Thirdly, fracture responses of the tibia subjected to a three point dynamic loading at $1.5 \mathrm{~m} / \mathrm{s}$ and vehicle impact loading at $11.1 \mathrm{~m} / \mathrm{s}$ were compared. The results were similar between the two speeds allowing for three point loading as a simplification for determining the failure load [9]. Similarly, the speed of a side kick at impact was found to be within a range of 5.2-6.9 m/s [19], which made findings from Mo et al. applicable here [9].

The simplifications listed above, i.e.: the tibia being modeled as a brittle cylindrical beam and a three-point dynamic bending load being applied, were used to determine the force required to break the tibial bone under bending by solving the equations for peak dynamic force acting on the distal tibia. Assuming that the force is applied in the distal third region of the tibia and using the length of the tibia, the location of the bone held in the three point bending test and the bending moment threshold determined from Kerrigan's model of 269 $\mathrm{Nm}$ [13], the applied force to cause a failure by bending was determined to be approximately $3500 \mathrm{~N}$. Applying the laws of trigonometry, if the applied impact in the form of a kick is oriented as described above, then approximately $4115 \mathrm{~N}$ is required for failure. Despite the limitations and unknowns as outlined in the introduction, the electromagnetic nature of the prototype made it possible to alter the force applied to cause a break to match a range of failure loads.

\section{CAD model}

Both the limb and the testing assembly were modeled in SolidWorks (Dassault Systems, SOLIDWORKS Corp, Massachusetts, USA) using material properties of American Iron and Steel Institute (AISI) 1018 
carbon steel. SolidWorks software was used to determine the center of gravity for the limb, visualize the deflection at the break location and the stress concentration of the model. It was also used to calculate the fatigue strength at the ball and socket joints in order to ensure the joints did not fail under repeated high loads. The upper and lower segments were joined together to make the model one continuous rigid body for the von Mises stress simulation and the deflection simulation. The ball on the lower limb was constrained and a static $3500-\mathrm{N}$ force perpendicular to the model at $157 \mathrm{~mm}$ from the ground was applied. The electromagnet, sockets and stands from the testing assembly were not taken into consideration in these simulations. Figure 2 is a photograph of the actual manufactured electromagnetic limb model (A) and the computer aided design (Solid Works) model representation of the limb (B).

\section{Electromagnetic model specifications and measurements}

The electromagnetic model as shown in Figure 2 (B) had a lower segment and an upper segment with the electromagnet mounted between to represent the entire human lower limb. Components were made using AISI 1215 carbon steel in order for the limb to be able to withstand forces greater than $3500 \mathrm{~N}$ of shear force. The break location was $157 \mathrm{~mm}$ above the ground hence the lower limb segment was also $157 \mathrm{~mm}$ in length as shown in Figure 3 (A). The electromagnetic model length was $496 \mathrm{~mm}$, which is identical to that of a $50^{\text {th }}$ percentile male tibial length of $402 \mathrm{~mm}$ with additional $94 \mathrm{~mm}$ ankle condyles depth to the ground [20]. This ensured that the model replicated a healthy human male standing prior to the impact. The electromagnetic model without its outer protective skin had a diameter of $88.9 \mathrm{~mm}$, which was smaller in diameter than the average male calf width of $110 \mathrm{~mm}$ [20]. This provided an additional $21.1 \mathrm{~mm}$ to add material replicating soft tissues for future development of the model. A stand was used to secure the limb at the top and bottom to provide a stable platform for dynamic testing. The characteristics of the model and those of a $50^{\text {th }}$ percentile human male lower limb are shown in Table 1.

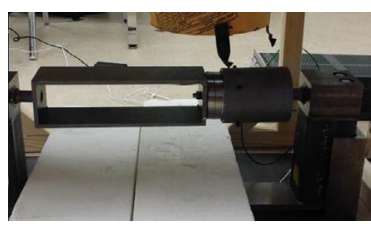

(A)

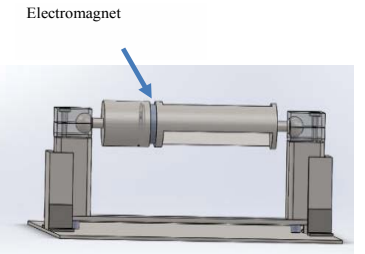

(B)
Figure 2: An actual photo of the electromagnetic model $(A)$ and the CAD representation of the electromagnetic model (B).

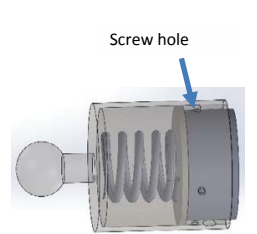

(A)

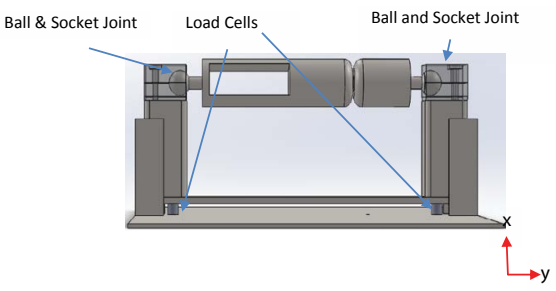

(B)
Figure 3: The lower limb segment of the electromagnet with the spring $(A)$ and the electromagnet assembly (B)

\section{Electromagnet}

In order to achieve the break force inflicted by a strike from any direction, a round electromagnet (APW Company, Rockway, New Jersey, USA) of $76.2 \mathrm{~mm}$ in diameter was used. A $76.2 \mathrm{~mm}$ electromagnet provides a maximum pull force of $1800 \mathrm{~N}$. Therefore, it was used in combination with a compression spring of $409780 \mathrm{~N} / \mathrm{m}$ of compression stiffness (McMaster Carr, Chicago, USA, \#96485K138) to provide the additional normal force to increase the required shear load prior to the breaking of the electromagnetic joint.

\section{Testing assembly}

A testing assembly was developed in order to safely and properly test the model. Figure 3 (A) shows the assembly of the spring and the electromagnet onto the lower segment of the limb. Set screws were inserted in the screw holes to ensure the magnet did not fall out of the housing. The shear strength of the screws was determined in order to ensure fatigue strength of over 1,000,000 cycles. The upper portion had a cut-out as seen in Figure 3 (B) to reduce the overall weight of the limb model and match the center of gravity of the $50^{\text {th }}$ percentile male lower limb. The limb segments were secured to the testing assembly via ball and socket joints that allowed the model to move along the $\mathrm{x}$-axis in a positive or negative direction as shown in Figure 3 (B).

\section{Dynamic testing}

A drop test was used to test the model dynamically. The drop height was determined based on the required kick speed, which was set between $5.2-6.87 \mathrm{~m} / \mathrm{s}$ as suggested by Wasik [19]. A drop height of $1.6 \mathrm{~m}$ was selected using a mean maximum velocity of $5.6 \mathrm{~m} / \mathrm{s}$. A different mass set at 450, 800, $10201140,1210,1260,1300$ and 1465 $\mathrm{g}$ was used for the drop test to incrementally increase the impact load while maintaining a constant impact velocity.

Two load cells (Omega Engineering Inc., Stamford, Connecticut, USA) were connected to a data acquisition system, which consisted of the CompaqDAQ chassis and a single four channel NI9215 module (National Instruments, Austin, Texas, USA) sampling force data from each channel at $100 \mathrm{kS} / \mathrm{sec}$. The module was connected to a computer via the universal serial bus. The computer had custom data collection software utilizing MATLAB and the Data Acquisition Toolbox (MathWorks, Matlab, Massachusetts, USA). The raw data was filtered using the Channel Frequency Class 1000 parameters in MATLAB with the Signal Processing Toolbox. Then the two load cell force traces were summed, the peak force was retained and the "break or no-break" outcome was recorded.

\section{Statistical analysis}

The binary logistic regression was used to obtain the break threshold values using Minitab 17 (Minitab Inc, Pennsylvania, USA). The logistic regression was run on the combined information of peak forces and binary ("break/ no break") data while the electromagnet was switched on Table 2. The two possible outcomes ("break/ no break") were then further analyzed by indicating the magnitudes of the chosen peak break forces that would predict the break threshold. The threshold in this case was defined as the magnitude of the peak break force in which $50 \%$ of the time a break would occur. In order to achieve this tolerance curve, a calculation of the logit was required and transformed into probability using equations 1 and 2 :

$$
\text { Equation } 1: t(p)=\beta_{0}+\beta_{1} X_{1}
$$


Equation 2: probability $=\frac{1}{1+e^{-\operatorname{logit}(\mathrm{p})}}$

where $\beta_{0}$ equals the logistic regression intercept and $\beta_{1}$ equals

the logistic regression coefficient for the first predicting variable $\left(X_{1}\right)$ $[21,22]$.

\section{Results}

The highest stress concentrations were observed at the necks on the balls of both the upper and lower segment (Figure $4 \mathrm{~A}$ and $\mathrm{B}$ ). The highest deflection of the limb, which was $0.28 \mathrm{~mm}$ along the line of applied load, occured where the two limb segments were joined together. While the electromagnet was switched on, the minimum force required to break the model, which meant separating the electromagnet and the lower segment from the upper segment, was $3237 \mathrm{~N}$ when a mass of $1.21 \mathrm{~kg}$ was dropped onto the model. While the electromagnet was switched off, the minimum force of $1423 \mathrm{~N}$ was required to break the model when a mass of $450 \mathrm{~g}$ was dropped (Table 2). Figure 5 shows the probability of obtaining a complete fracture based on the peak break force of the limb, where $\beta 0=-2.54$ and $\beta 1=0.000613$. The threshold was estimated by finding the $50 \%$ probability magnitude of the break force (x-axis). The $50 \%$ probability of fracture corresponded to a force of $4144 \mathrm{~N}$.

\section{Discussion}

The purpose of the present study was to design, manufacture and test an artificial human limb model with similar characteristics as that of a 50th percentile male lower limb, which could be used for compound fracture simulations at a desired location and by a desired shear force. The developed prototype of a breakable and reusable human lower limb model is first of its kind and it enabled us to test the feasibility of replicating a compound lower limb fracture from specific strikes. The dynamic testing results showed that the minimum force required to break the electromagnetic limb resulting in a complete separation of the electromagnet when it was switched on and the lower limb segment from the upper limb segment was $3237 \mathrm{~N}$. This result was surprising as the maximum pull force that the electromagnet was capable of providing was $1800 \mathrm{~N}$. The addition of the compression spring provided the additional normal force to increase the required shear load. The $50 \%$ probability of fracture occurrence was at 4144 $\mathrm{N}$ (Figure 5), which was greater than the required $3500 \mathrm{~N}$ force as calculated in the methods section of the manuscript. The characteristics of the prototype as shown in Table 1 correspond well with a $50 \%$ male lower limb, as well as a range of limb sizes both above and below this size. Therefore, the prototype proved to be capable of breaking with the same force magnitude as a real human lower limb would. However, the oversimplifications discussed have to be adressed before future development of the model and its application in other domains.

\section{Other commercially available models and methods}

Other manufacturers have worked on increasing the biofidelity of existing lower limb models that are used to detect potential injury. The most widely used model is the Hybrid III Anthropomorphic Test Dummy (ATD) (Humanetics Innovative Solutions Inc, Plymouth, Michigan, U.S.A.). While this model is the current crash testing standard and has good instrumentation capability, recent revisions focus on improving biofidelity in the lower leg, ankle, and foot. The ATD can also be used in many non-automotive applications such as

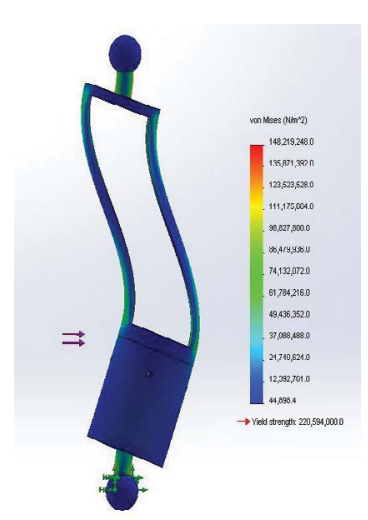

(A)

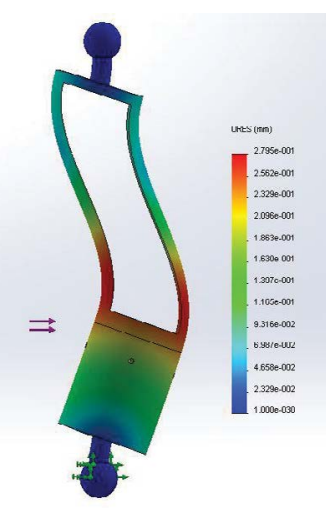

(B)

Figure 4: Von Mises stress analysis of the electromagnetic limb $(A)$ and the deflection of electromagnetic limb (B)

\begin{tabular}{|c|c|c|c|}
\hline $\begin{array}{l}\text { Electromagnet } \\
\text { Engagement* }\end{array}$ & Mass $(\mathbf{k g})$ & Peak Break Force (N) & Break as a Result? \\
\hline No & 0.450 & 1423 & Yes \\
\hline No & 0.800 & 3546 & Yes \\
\hline Yes & 0.450 & 276 & No \\
\hline Yes & 0.450 & 298 & No \\
\hline Yes & 0.800 & 3816 & No \\
\hline Yes & 0.800 & 4709 & No \\
\hline Yes & 1.020 & 4247 & No \\
\hline Yes & 1.020 & 4970 & No \\
\hline Yes & 1.140 & 3923 & No \\
\hline Yes & 1.140 & 4458 & Yes \\
\hline Yes & 1.140 & 5047 & No \\
\hline Yes & 1.210 & 3237 & Yes \\
\hline Yes & 1.210 & 5556 & Yes \\
\hline Yes & 1.210 & 5012 & Yes \\
\hline Yes & 1.210 & 4350 & Yes \\
\hline Yes & 1.263 & 4769 & Yes \\
\hline Yes & 1.300 & 3275 & Yes \\
\hline Yes & 1.470 & 6394 & Yes \\
\hline
\end{tabular}

Table 2: Dynamic loading test results of the electromagnet breaking mechanism. * The engagement of the electromagnet means whether the magnet was switched on or off: "yes" means it was switched on, "no" means it was switched off.

sports equipment testing. However, the long bones of the ATD lower limb are rigid and do not allow for fracture dynamics testing unlike our prototype. An improvement on this was developed by the Japan Automotive Research Institute in 2003. Their model was called the Flexible Pedestrian Legform Impactor (Flex-PLI), in which a femur and lower limb model could simulate the human bone flexibility and knee joint stiffness properly. However, the Flex-PLI similarly to the ATD is not actually capable of "breaking" or simulating a compound human lower limb fracture (even though it can predict it) unlike our prototype. Therefore, our model is superior to both the ATD and FlexPLI for the purposes of fracture simulations. Similarly to the aim of our study, finite element (FE) methods could also be used for simulations of human bone fractures. Wong et al. (2010) used FE methods to simulate fracture mechanisms at the tibia, obtained adequate fracture locations and an indication of the fracture morphology in agreement to clinical 


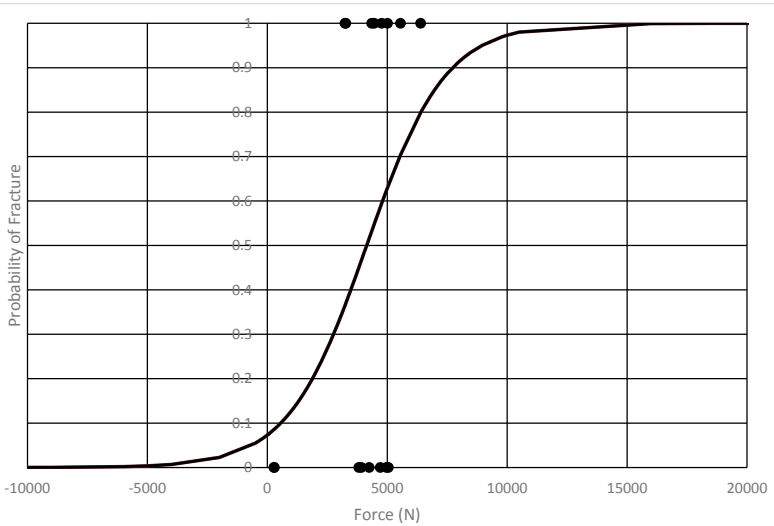

Figure 5: The probability of a fracture represented by the solid line [y-axis] versus the peak break force $[\mathrm{x}$-axis]. Binary break data are shown in black (closed circles).

data [6]. Apart from assessing the bending load as this study did, the authors also analysed torsional and axial load, and included fibula in their model [6]. However, bending load as investigated here was shown to be responsible for the majority of tibial fractures regardless of the fracture site, with the distal third part of the tibia being the most common fracture location based on automobile crashes analysis [11].

\section{Limitations and recommendations}

While the present model has the capability of providing a range of break forces, lower limb fracture has yet to be investigated under dynamic loading conditions similar to a kick. Therefore, it is important that additional experiments are carried out to confirm the break location and break force proposed here. These further experiments could be done using our prototype, as well as by doing tests on cadavers or by using methods of FE Models. Three-dimensional FE analysis was used to evaluate stresses and displacements of the tibia bone under physiological loading and the results showed that it was an appropriate method in finding the causes of long-term bone fracture for clinical practice [5]. While the FE methods might provide a foundation for further studies of bone injury prevention, bone transplant and subject-specific fracture mechanism [5], our prototype can provide an actual training platform for specific loads at specific locations. Both methods could complement each other and be used together to investigate the developed stresses at the tibia under both physiological and pathological loading in order to determine the response of the bone. There is a number of limitations in our study. Firstly, the assumption and simplification of modeling the tibia as a cylindrical beam. This could be potentially addressed in the next stage of the present model development. Secondly, the system used for the testing needs to be validated against other methods available for fracture simulation research. Thirdly, while tibial geometry was oversimplified in the present study, we also did not include the fibula in our model. Despite the fact that the distal tibial fracture is the most common [11], the fibula contributes to the structure and strength of the lower limb and therefore future prototypes would benefit from its inclusion in the model. Furthermore, we only tested the prototype to fail under bending force, not addressing axial or torsion forces. However, as mentioned before it appears that bending is responsible for the majority of tibial fractures regardless of the fracture site [11]. Lastly, we excluded the role of lower limb muscles and their contribution to such fractures, and so their effect remains unknown. Similarly, the tibiofibular joints and interosseous membrane were not simulated in our prototype. We acknowledge that the above mentioned limitations would need to be addressed in future model development in order to get more accurate results comparable to human anatomy. Furthermore, the present prototype can be developed to create an upright model of the human lower limb to be used for applications in sports and self-defense, military, police or medical industries. Although it is unknown how the addition of a skin and soft tissue analog would change the model break thresholds, it is clear that the prototype can break at various loads and could be modified for various applications and further fracture dynamics research.

\section{Conclusion}

A prototype model for testing the feasibility of replicating a compound human lower limb fracture from specific strikes has been developed. The prototype had realistic dimensions, fracture locations and load to fracture magnitudes of a $50^{\text {th }}$ percentile human male lower limb. The model is reusable and therefore suitable for repeated testing. This paper presents the characteristics of the model, results of the break under similar forces that cause real-life human lower limb compound fractures and recommendations for the future development of the model.

\section{Acknowledgements}

The authors would like to thank Mr. Tim Larkin from Target Focus Training Group for funding this important research and providing the authors with selfdefense technique expertise, unfettered discretion in constructing the prototype designing the test methodology and reaching their conclusions. Furthermore, the authors extend their gratitude to Ms. Katerina Doslikova, Ms. Christina M. Stevens and Mr. Mark Nolette all of GTD Engineering.

\section{Conflict of interest statement}

There is no conflict of interest to report. Besides the contract between GTD Engineering and Target Focus Training Group to produce third party results, the authors have no affiliations with or involvement in any organization or entity with any financial interest (stock ownership or patent-licensing arrangements), or nonfinancial interest in the subject matter or materials discussed in this manuscript.

\section{References}

1. Chomiak J, Junge A, Peterson L, Dvorak J (2000) Severe injuries in football players. Influencing factors. Am J Sports Med 28: S58-S68.

2. Goga I, Gongal $P$ (2003) Severe soccer injuries in amateurs. Br J Sports Med 37: 498-501.

3. Birrer RB (1996) Trauma epidemiology in the martial arts. The results of an eighteen-year international survey. Am J Sports Med 24: S72-S79.

4. Shepherd JP, Shapland M, Pearce NX, Scully C (1990) Pattern, severity and aetiology of injuries in victims of assault. J R Soc Med 83: 75-78.

5. Phate N, Nareliya R, Kumar V, Francis A (2014) Three-Dimensional Finite Element Analysis of Human Tibia Bone. IJSRET 3: 52-56.

6. Wong C, Mikkelsen P, Hansen LB, Darvann T, Gebuhr P (2010) Finite element analysis of tibial fractures. Dan Med Bull 57: A4148.

7. Desmoulin GT, Larkin T (2012) Effectiveness of slow rate practice techniques. 1-10.

8. Otte D, Haasper C (2007) Characteristics on fractures of tibia and fibula in car impacts to pedestrians and bicyclists - Influences of car bumper height and shape. Annu Proc Assoc Adv Automot Med 51: 63-79.

9. Mo F, Arnoux PJ, Jure JJ, Masson C (2012) Injury tolerance of tibia for carpedestrian impact. Accid Anal Prev 46: 18-25.

10. Chang WR, Kapasi Z, Daisley S, Leach WJ (2007) Tibial shaft fractures in football players. J Orthop Surg Res 2: 11.

11. Ivarsson BJ, Manaswi A, Genovese D, Crandall JR, Hurwitz SR, et al. (2008) Site, type, and local mechanism of tibial shaft fracture in drivers in frontal automobile crashes. Forensic Sci Int 175: 186-192. 
Citation:Desmoulin GT, Rashid FAN, Ropchan C, Meitz J, Upadhyaya K, et al. (2015) A New Approach to Fracture Simulations: The Electromagnetic Breakable and Reusable Human Lower Limb Model. J Forensic Biomed 4: 121. doi: 10.4172/2090-2697.1000121

12. Hoffmeister BK, Smith SR, Handley SM, Rho JY (2000) Anisotropy of Young's modulus of human tibia cortical bone. Med Biol Eng Comput 38: 333-338.

13. Kerrigan JR, Parent DP, Untaroiu C, Crandall JR, Deng B (2009) A new approach to multibody model development: Pedestrian lower extremity. Traffic Inj Prev 10: 386-397.

14. Yamada $H$ (1970) Strength of biological materials. Williams \& Wilkins, Baltimore.

15. Cerritelli B, Maruff $P$, Wilson $P$, Currie $J(2000)$ The effect of an external load on the force and timing components of mentally represented actions. Behav Brain Res 108: 91-96.

16. Fontani G, Migliorini S, Benocci R, Facchini A, Casini M, et al. (2007) Effect of mental imagery on the development of skilled motor actions. Percept Mot Skills 105: 803-826.

17. Pheasant ST (1986) Bodyspace: Anthropometery, ergonomics, and design Taylor \& Francis, London, Philadelphia.
18. Ebacher V, Tang C, McKay H, Oxland TR, Guy P, et al. (2007) Strain redistribution and cracking behaviour of human bone during bending. Bone 40 1265-1275.

19. Wasik J (2011) Kinematics and kinetics of taekwon-do side kick. J Hum Kinet 30: $13-20$.

20. Kuppa S, Wang J, Haffner M, Eppinger R (2001) Lower extremity injuries and associated injury criteria. National Highway Safety Administration, USA, Paper No. 457.

21. Desmoulin GT, Anderson GS (2011) Method to investigate contusion mechanics in living humans. J Forensic Biomech 2: 1-10.

22. Hardy BJ, Lawrence GJL, Knight IM, Simmons ICP, Carroll JA et al. (2007) A study of possible future developments of methods to protect pedestrians and other vulnerable road users. Project Report UPR/VE/061/07 forthe European Commission, Directorate-General Enterprise and Industry, Automotive Industry. 